\title{
Cholecystocutaneous Fistula following Drainage of Parietal Abscess: A Rare Case Report
}

\author{
MAA AMIN ${ }^{\mathrm{a}}$, ME ULLAH ${ }^{\mathrm{b}}$, N SHABNAM ${ }^{\mathrm{c}}$
}

Summary:

Spontaneous perforation of gallbladder as a complication of biliary stones may lead to cholecystocutaneous abscess or fistula. Here we report a case of cholecystocutaneous fistula in a 50-year-old diabetic female patient who presented with a chronic discharging sinus on right upper abdomen

\section{Introduction:}

Most cholecystocutaneous fistulae are postoperative complications of liver and biliary tract surgery or trauma. External biliary fistulae rarely occur spontaneously as a result of intrahepatic abscess, necrosis or perforation of the gallbladder, or other inflammatory process involving the biliary tree ${ }^{1}$ Spontaneous cholecystocutaneous fistulae are often the result of neglected biliary tract disease. This is rarely observed today because of the early diagnosis and management made possible by ultrasonography, broad-spectrum antibiotics, and effective surgical management of biliary tract disease ${ }^{2}$. The pathophysiology of this condition has been associated with increased pressure in the gallbladder, secondary to biliary obstruction. Ultrasonography or computed tomography and fistulography play an important role in the diagnosis of cholecystocutaneous fistula. The treatment of such fistula requires elective cholecystectomy with excision of the fistula tract and drainage of any associated abscess with adequate antibiotic coverage.

a. Dr. Md. Abdullah-Al-Amin, Professor \& Head, Department of Surgery \& MISC, BIRDEM \& Ibrahim Medical College, Dhaka, Bangladesh.

b. Dr. Mahmud Ekram Ullah, Assistant Professor, Dept of Surgery \& MISC, BIRDEM \& Ibrahim Medical College, Dhaka, Bangladesh.

c. Dr. Nilufar Shabnam, Registrar, Dept of Surgery \& MISC, BIRDEM \& Ibrahim Medical College, Dhaka, Bangladesh.

Address of Correspondence: Dr. Md. Abdullah -Al -Amin, Professor \& Head of the Department, Surgery \& MISC, BIRDEM \& Ibrahim Medical College, Dhaka, Bangladesh, Email: alamin.abdullah00@gmail.com

Received: 29 January, 2013

Accepted: 23 August, 2013 with recurrent abscess formation which failed to heal despite repeated attempts at incision drainage and debridement. After evaluation the tract was explored and was found to be communicating with the fundus of the gall bladder. The whole fistula tract was excised along with cholecystectomy.

(J Banagladesh Coll Phys Surg 2014; 32: 37-40)

\section{Case report:}

Mrs. X, a 50 year old diabetic lady hailing from Comilla got admitted in BIRDEM Hospital on 22/11/2010 with a chronic discharging sinus on right upper abdomen for 8 months. She had a history of drainage of parietal abscess over the same area 9 months back followed by secondary closure of the wound. But she repeatedly developed wound infection afterwards and the wound never really healed completely. She noticed recurrent seropurulent discharge through the central part of the scar. She had no history of nausea, vomiting, anorexia, fatty food intolerance, and fever or weight loss.

On examination, a drawn in scar $(8-10 \mathrm{~cm})$ was noted in the right hypochondrium with a small opening in the centre. On gentle compression, seropurulent discharge came out through the opening. Surrounding tissue was indurated but non-tender and local temperature was not raised. Abdominal and other systemic examination were unremarkable.

Our first clinical impression was tubercular sinus. The wound swab microscopy did not reveal any AFB. However, exploration of the tract was needed anyway and we planned to send the tract / tissue scrap for histopathological examination. MT or other serological tests were deemed unnecessary in this case.

Her other baseline hematological and biochemical investigations were all within normal limit. Wound Swab for C/S reported no growth. USG of abdomen demonstrated irregular hypoechoic area in muscle layer suggesting collection. However, it also showed sludge in gall bladder lumen (Fig-I). Sinogram revealed a linear tract and the contrast medium passed into a cavity (Fig-II).

Under G/A the sinus tract was explored by excising the previous scar and some seropurulent collection in the 
subcutaneous space was drained. On further exploration, it was found to be communicating with an intraabdominal viscus. So the incision was extended and deepened as right subcostal incision through which abdominal cavity was explored. The fistula tract was found connected to the fundus of the gall bladder. Gall bladder was found adherent to the parietes, filled with pus and a stone was impacted in the cystic duct. The whole fistula tract was excised along with cholecystectomy. Her post-operative period was uneventful.

Histopathology of gall bladder reported as acute on chronic cholecystitis and tissue from fistula tract showed infiltration of mostly chronic and also acute inflammatory cells with in the wall of the tract. No granuloma or malignancy was seen. So our final diagnosis was chronic empyema of the gall bladder with spontaneous cholecystocutaneous fistula.

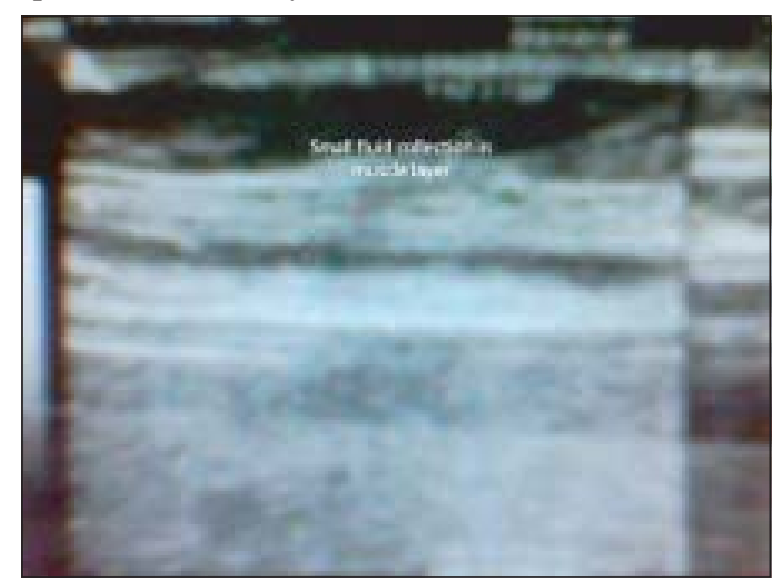

Fig-1: USG showing irregular hypoechoic area in muscle layer

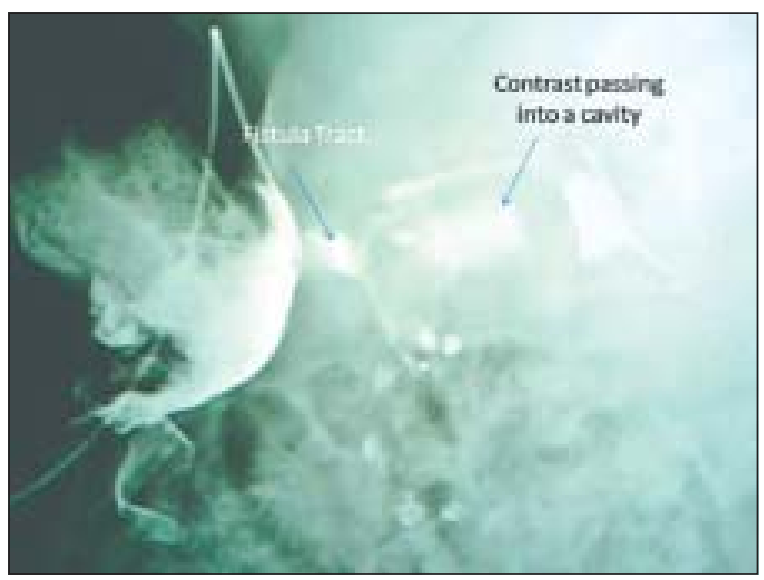

Fig-2: Contrast $X$-ray delineating the tract and a cavity

\section{Discussion:}

Spontaneous cholecystocutaneous abscess or fistula is an uncommon complication of gallbladder disease that has been known since the time of Thilesius in 1670 . Courvoisier documented 499 cases of gallbladder perforation in the late 19th century; 169 of these cases formed cutaneous tracts ${ }^{3}$. Over the past century, there have been fewer accounts of this problem because of prompt and safe management of biliary tract disease. In their review in 1949, Henry and Orr found 36 cases of external biliary fistulae reported after $1890^{3}$. In 2004, Vasanth A, Siddiqui A and O'Donnell K. have reported 20 cases over 'past 50 years'2.

External biliary fistulae or abscesses rarely occur spontaneously following necrosis or perforation of the gallbladder, or other inflammatory process involving the biliary tree. In the past, external drainage of the abscess and antibiotics were used for sepsis control. However, this approach has been associated with biliary fistula formation. Most cholecystocutaneous abscesses or fistulae are however iatrogenic or traumatic ${ }^{4}$.

In a review in 1994, Kaminsky reported on the frequency of biliary fistula. In this series, cholecystocutaneous abscesses or fistulae accounted for only $2 \%$ of all the cases $^{4}$. Generally, the fistulae or abscesses appear in the right upper quadrant, although other locations such as the epigastrium, umbilical area, right groin and even the gluteal region have also been described ${ }^{5}$.

Spontaneous cholecystocutaneous fistulas are almost always a result of neglected biliary tract disease ${ }^{2}$. Patients with this complication usually do not have classic symptoms normally associated with cholecystitis in their history, since this would have brought such a patient to seek medical attention sooner. Their symptoms tend to be more non-specific and often masked by coexistent disease. Symptoms of underlying disease may be ignored by the patient or may be masked by some underlying neuropathy ${ }^{6}$. This is consistent with the presentation in our case. Apart from the complaints of repeated local pain and swelling during episodes of infection, she did not have the classic symptoms of cholecystitis.

The patients are usually women over the age of $60^{7}$. However, cases have been documented in patients as 
young as 24 years old. The fistula usually presents itself as an enlarging mass before spontaneous rupture. In some cases, it may be associated with symptoms of upper abdominal colic, dyspepsia, jaundice, or weight loss ${ }^{6}$.

The pathophysiology of this condition has been associated with increased pressure in the gallbladder, secondary to cystic duct obstruction, most commonly due to calculi and rarely due to gallbladder carcinoma ${ }^{5}$. The increase in intraluminal pressure leads to impairment of the blood flow to and lymph drainage from the gallbladder, thus causing mural necrosis and perforation. Subacute perforation results in an abscess formation around the gallbladder with the formation of an internal or external biliary fistula. These fistulas, as presented in this case, frequently arise from the fundus of the gallbladder. The state preceding spontaneous rupture has been termed "empyema necessitatis" by Nayman ${ }^{8}$. This term essentially describes a "burrowing abscess" of the abdominal wall as a result of gallbladder inflammation.

The external opening of the fistula can be confused with a pyogenic granuloma, infected epidermal inclusion cyst, or metastatic carcinoma ${ }^{6}$. The discharge from the fistula may be purulent and mucoid if the cystic duct is obstructed by a stone as was found in our case. In cases where the cystic duct remains patent, discharge may be bilious. Intraoperatively the gallbladder usually appears small, contracted, chronically inflamed, and adherent to the parietes ${ }^{7}$.

Our patient had no particular symptoms attributable to gall bladder disease. Even though her gall bladder was filled with pus, she was otherwise asymptomatic. Chronic empyema is such a condition that is sometimes seen particularly in diabetic elderly population where clinical manifestation of inflammatory response is subdued. Advancing age, diabetic autonomic neuropathy and sub-optimal use of antibiotics may be an explanation. In their large study comprising 1392 patients having gallbladder disease, Thornton $\mathrm{J} \mathrm{R}$ and his colleagues showed that empyema of the gallbladder is sometimes chronic, painless, and afebrile. ${ }^{9}$

Imaging plays an important role in the diagnosis of this complication. Before fistula formation, the abscess can be diagnosed via ultrasonography, with findings that include a sonolucent mass with echogenic material adjacent to the anterior abdominal wall ${ }^{10}$. Sino/ fistulogram, allows visualization of its origin and course $^{5}$. Ultrasonography and CT imaging can also help in the diagnosis of this complication.

Management of cholecystocutaneous fistula should initially include control of any acute inflammatory process. This can be done by incision and drainage of the abscess if any, followed by wound cultures and appropriate antibiotic therapy. However, no bacteria could be isolated in our case. Surgically, the fistula tract can be laid open, with removal of any gallstones present in poor risk patient ${ }^{11}$. Spontaneous healing of cholecystocutaneous fistula has been reported in some cases $^{3}$. However standard treatment is elective cholecystectomy along with excision of the fistula tract and drainage of any associated abscess.

\section{Conclusion:}

This case report demonstrates that maintaining a high degree of suspicion of this rare entity is helpful during surgery. The possibility of cholecystocutaneous fistula should be considered in any patient who has a discharging sinus in the right upper abdominal or chest wall. Early cholecystectomy and excision of the fistula tract can reduce morbidity from this complication. However, continued advances in noninvasive investigations, and widespread practice of elective and emergency cholecystectomy will eventually put an end to this exceedingly rare complication of common gallbladder disease.

\section{References:}

1. Sodhi K, Athar M. Spontaneous Cholecysto-Cutaneous Fistula Complicating Carcinoma of the Gall Bladder: A Case Report. Indian Journal of Surgery. April 2012; 74 (2): 191-193.

2. Vasanth A, Siddiqui A, O’Donnell K. Spontaneous cholecystocutaneous fistula. South Med J. 2004;97:183-5

3. Henry CL, Orr TG Jr. Spontaneous external biliary fistulas. Surgery 1949; 26: 641-646.

4. Kaminski DL. Clinical syndromes involving the gallbladder. In: Braasch JW, Tompkins RK, editors. Surgical disease of the biliary tract and pancreas. Philadelphia: Mosby; 1994. 115-28.

5. Nicholson T, Born MW, Garber E. Spontaneous cholecystocutaneous fistula presenting in the gluteal region. J Clin Gastroenterol. 1999; 28(3):276-277 
6. Hoffman L, Beaton H, Wantz G. Spontaneous cholecystocutaneous fistula: A complication of neglected biliary tract disease. J Am Geriatr Soc. 1982; 30: 632-634.

7. Rosario P, Gerst P, Prakash K, et al. Cholecystocutaneous fistula: An unusual presentation. Am J Gastroenterol. 1990; 85: 214-215.

8. Nayman J. Empyema necessitatis of the gall-bladder. Med J Aust. 1963; 1:429-430
9. Thornton J R, Heaton K W, Espiner H , et al. Empyema of the gall bladder - reappraisal of a neglected disease. Gut. 1983;24:1183-1185

10. Ulreich S, Henken EM, Levinson ED. Imaging in the diagnosis of cholecystocutaneous fistulae. J Can Assoc Radiol. 1983; 34: 39-41.

11. Gibson TC, Howat JM. Cholecystocutaneous fistula. Br J Clin Pract. 1987; 41: 980-982. 